\title{
Frustration induced disordered magnetism in $\mathrm{Ba}_{3} \mathrm{RuTi}_{2} \mathrm{O}_{9}$
}

\author{
Tusharkanti Dey and A.V. Mahajan \\ Department of Physics, Indian Institute of Technology Bombay, Powai, Mumbai 400076, India
}

\section{Abstract}

The title compound $\mathrm{Ba}_{3} \mathrm{RuTi}_{2} \mathrm{O}_{9}$ crystallizes with a hexagonal unit cell. It contains layers of edge shared triangular network of $\mathrm{Ru}^{4+}(S=1)$ ions. Magnetic susceptibility $\chi(T)$ and heat capacity data show no long range magnetic ordering down to $1.8 \mathrm{~K}$. A Curie-Weiss $(\mathrm{CW})$ fitting of $\chi(T)$ yields a large antiferromagnetic CW temperature $\theta_{\mathrm{CW}}=-166 \mathrm{~K}$. However, in low field, a splitting of zero field cooled (ZFC) and field cooled (FC) $\chi(T)$ is observed below $\sim 30 \mathrm{~K}$. Our measurements suggest that $\mathrm{Ba}_{3} \mathrm{RuTi}_{2} \mathrm{O}_{9}$ is a highly frustrated system but only a small fraction of the spins in this system undergo a transition to a frozen magnetic state below $\sim 30 \mathrm{~K}$.

PACS numbers: 75.47.Lx, 75.50.Lk, 75.40.Cx

\section{INTRODUCTION}

In recent times, $4 d$ and $5 d$ transition metal based materials have been in the focus as their driving physics is different from their $3 d$ counterparts but have not been explored much. This variation arises because of their extended ' $d$ ' orbitals, a small onsite Coulomb energy ' $U$ ', and a large spin-orbit coupling (SOC) [1]. The interplay between $\mathrm{U}$ and SOC makes these materials interesting. Many systems of the $4 d / 5 d$ family like $\mathrm{Sr}_{2}(\mathrm{Ir} / \mathrm{Ru} / \mathrm{Rh}) \mathrm{O}_{4}$ [1] 3], $\mathrm{Ca}_{2} \mathrm{RuO}_{4}$ [4], $\mathrm{Na}_{2} \mathrm{IrO}_{3}$ [5], $\mathrm{Na}_{4} \mathrm{Ir}_{3} \mathrm{O}_{8}$ [6], $(\mathrm{Sr} / \mathrm{Ca})_{3} \mathrm{Ru}_{2} \mathrm{O}_{7}$ [7, 8] etc. have drawn attention in recent years.

Recently, we reported results of our investigations of hexagonal $\mathrm{Ba}_{3} \mathrm{IrTi}_{2} \mathrm{O}_{9}$ with $5 d \mathrm{Ir}^{4+}$ (possibly $J_{\text {eff }}=1 / 2$ ) ions forming a $2 \mathrm{D}$ triangular network [9]. A large sitedisorder between $\mathrm{Ir}^{4+}$ and $\mathrm{Ti}^{4+}$ cations plays an important role in the magnetic properties of the sample. The system remains paramagnetic down to $0.35 \mathrm{~K}$ despite a large antiferromagnetic (AF) Curie-Weiss temperature $\theta_{\mathrm{CW}} \sim-130 \mathrm{~K}$. Magnetic heat capacity at low temperature follows a power law with temperature. These results suggested that $\mathrm{Ba}_{3} \mathrm{IrTi}_{2} \mathrm{O}_{9}$ is in a spin liquid ground state with $5 d \operatorname{Ir}^{4+}$ (possibly $J_{\text {eff }}=1 / 2$ ) in a $2 \mathrm{D}$ triangular network. The SOC presumably plays a significant role in determining the properties of the system. It will be interesting to study its $4 d$-analog (say, based on $\mathrm{Ru}$ ) where spin-orbit coupling effect is expected to be of intermediate strength compared to that in $3 d$ and $5 d$ elements.

Dickson et al. [10] measured the magnetic susceptibility variation with temperature of hexagonal $\mathrm{Ba}_{3} \mathrm{RuTi}_{2} \mathrm{O}_{9}$ in the limited temperature range $253 \mathrm{~K}$ to $323 \mathrm{~K}$. Susceptibility data in a larger temperature range $77-335 \mathrm{~K}$ are reported in Ref. [11]. In both the papers, a high, AF $\theta_{\mathrm{CW}}$ $(\sim-250 \mathrm{~K})$ is inferred [10, 11]. These reports indicate that $\mathrm{Ba}_{3} \mathrm{RuTi}_{2} \mathrm{O}_{9}$ could also be a frustrated spin liquid candidate and a $4 d$ analog of $\mathrm{Ba}_{3} \mathrm{IrTi}_{2} \mathrm{O}_{9}$ with $S=1$. Thus low temperature measurements on $\mathrm{Ba}_{3} \mathrm{RuTi}_{2} \mathrm{O}_{9}$ are warranted to confirm its magnetic ground state.

*Email: tusdey@gmail.com
In this paper we report preparation, structural analysis, magnetic susceptibility and heat capacity measurements on $\mathrm{Ba}_{3} \mathrm{RuTi}_{2} \mathrm{O}_{9}$. The system crystallizes in the space group $\mathrm{P} 6{ }_{3} \mathrm{mc}$ with a large site sharing between $\mathrm{Ru}^{4+}$ and $\mathrm{Ti}^{4+}$ ions as reported earlier [12] and similar to that in $\mathrm{Ba}_{3} \mathrm{IrTi}_{2} \mathrm{O}_{9}$ [9]. Susceptibility data show no long range ordering down to $2 \mathrm{~K}$ but splitting between zero field cooled (ZFC) and field cooled (FC) data is found below $30 \mathrm{~K}$. A large $\theta_{\mathrm{CW}}(\mathrm{AF})$ obtained from $\mathrm{CW}$ fitting indicates strong correlation between the magnetic ions. No anomaly, even at $30 \mathrm{~K}$, is found in heat capacity measurements down to $1.8 \mathrm{~K}$. These measurements suggest that the system is highly frustrated and perhaps a fraction of the spins form a disordered magnetic state below $30 \mathrm{~K}$.

\section{EXPERIMENTAL DETAILS}

A polycrystalline sample of $\mathrm{Ba}_{3} \mathrm{RuTi}_{2} \mathrm{O}_{9}$ was prepared by conventional solid state reaction method using high purity $\mathrm{BaCO}_{3}, \mathrm{TiO}_{2}$ and $\mathrm{Ru}$ metal powder as starting materials. Stoichiometric amount of the starting materials were mixed thoroughly, pressed into a pellet and calcined at $900^{\circ} \mathrm{C}$ for $15 \mathrm{~h}$. After calcination, the pellet was crushed into powder, pelletized and fired at $1100^{\circ} \mathrm{C}$ for $100 \mathrm{~h}$ with several intermediate grindings.

Powder x-ray diffraction (XRD) measurements were performed at room temperature with $\mathrm{Cu} K_{\alpha}$ radiation $(\lambda=1.54182 \AA)$ in a PANalytical X'Pert PRO diffractometer using $\mathrm{Si}$ for calibration. Magnetization $M$ measurements were carried out in the temperature $T$ range $2-400 \mathrm{~K}$ using a Quantum Design PPMS and SQUID VSM. Heat capacity measurements were done in the temperature range $1.8-300 \mathrm{~K}$ and field $H$ range $0-9 \mathrm{~T}$ using the heat capacity attachment of Quantum Design PPMS.

\section{RESULTS AND DISCUSSION}

Single phase nature of our sample was confirmed from the XRD measurements. Maunders et al. have reported $\mathrm{Ba}_{3} \mathrm{RuTi}_{2} \mathrm{O}_{9}$ to crystallize in the space group $\mathrm{P} 6_{3} m c$ [13]. 

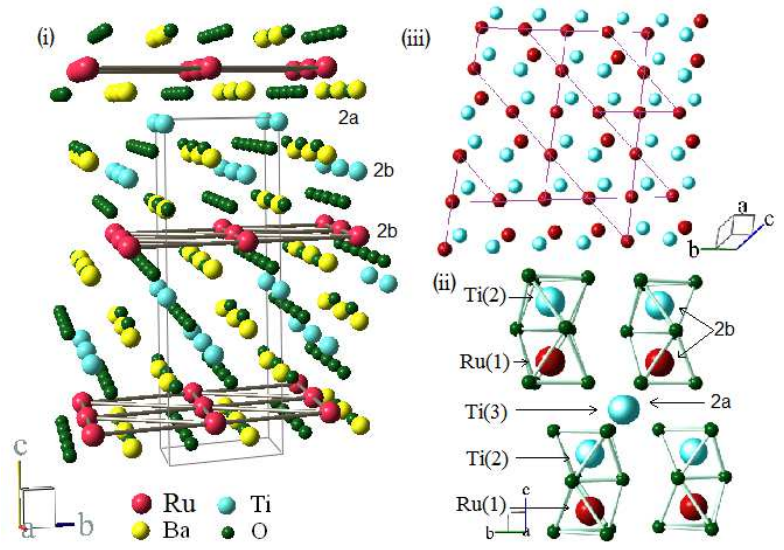

Figure 1: (i) The ideal structure of $\mathrm{Ba}_{3} \mathrm{RuTi}_{2} \mathrm{O}_{9}$ without any site disorder between $\mathrm{Ti}^{4+}$ and $\mathrm{Ru}^{4+}$ ions is shown. The $\mathrm{Ru}^{4+}$ spins form an edge shared triangular network parallel to the $a b$ plane as shown. (ii) The $\mathrm{RuTiO}_{9}$ bioctahedra are shown (iii) A possible arrangement of $\mathrm{Ti}^{4+}$ and $\mathrm{Ru}^{4+}$ ions in the $a b$ plane is shown when about $33 \%$ of $\mathrm{Ru}^{4+}$ ions exchange their positions with $\mathrm{Ti}^{4+}$ ions in the $2 \mathrm{~b}$ site.

In this space group, the $2 \mathrm{a}$ site is occupied by the $\mathrm{Ti}^{4+}$ ions and in the $2 \mathrm{~b}$ sites $\mathrm{Ti}^{4+} / \mathrm{Ru}^{4+}$ ions reside in an ordered manner. In the ideal case, the ordered arrangement of $\mathrm{Ti}^{4+}$ and $\mathrm{Ru}^{4+}$ ions form face-sharing $\mathrm{RuTiO}_{9}$ bioctahedra (Fig. 1(ii)) and the $\mathrm{Ru}^{4+}$ spins form an edge-shared triangular network in the $a b$ plane (as shown in Fig. [(i)). However, a site sharing between $\mathrm{Ti}^{4+}$ and $\mathrm{Ru}^{4+}$ ions is expected as the two ions are of similar ionic size. In Ref. [12] four possible arrangements of $\mathrm{Ru}^{4+} / \mathrm{Ti}^{4+}$ ions have been suggested in $\mathrm{Ba}_{3} \mathrm{RuTi}_{2} \mathrm{O}_{9}$. Among them, the two arrangements where $\mathrm{RuTiO}_{9}$ and $\mathrm{Ru}_{2} \mathrm{O}_{9} / \mathrm{Ti}_{2} \mathrm{O}_{9}$ bioctahedra are formed have been found to be energetically more probable [12]. We refined our $\mathrm{XRD}$ data using the space group $\mathrm{P} 6{ }_{3} m c$ and considering a site sharing between $\mathrm{Ti}^{4+}$ and $\mathrm{Ru}^{4+}$ ions. We have found almost $31 \%$ site exchange of $\mathrm{Ru}^{4+}$ ions with $\mathrm{Ti}^{4+}$ ions in the $2 \mathrm{~b}$ site and an $8 \%$ site sharing with $2 \mathrm{a}$ site $\mathrm{Ti}^{4+}$ ions. This situation is very similar to that found in Ref. [12]. In $\mathrm{Ba}_{3} \mathrm{IrTi}_{2} \mathrm{O}_{9}$ as well, we found a large site sharing of $\mathrm{Ir}^{4+}$ ions with $\mathrm{Ti}^{4+}$ ions both in $2 \mathrm{~b}$ as well as 2a sites [9].

Fig. 2 shows the XRD refinement pattern which yields $\mathrm{R}_{p}=5.7 \%, \mathrm{R}_{w p}=7.7 \%$ and goodness of fit $(\mathrm{GOF})=9.70$. The lattice parameters obtained from the refinement are shown in Table $\square$ and compared with the values with $\mathrm{Ba}_{3} \mathrm{RuTi}_{2} \mathrm{O}_{9}$ (reported earlier), $\mathrm{Ba}_{3} \mathrm{IrTi}_{2} \mathrm{O}_{9}$ and $\mathrm{BaTiO}_{3}$ (parent compound). Values obtained by us are in good agreement with others. The atomic positions and occupancies resulting from our refinement are shown in Table II

As a result of site sharing between $\mathrm{Ti}^{4+}$ and $\mathrm{Ru}^{4+}$ ions, the $\mathrm{Ru}^{4+}(S=1)$ edge shared triangular plane will be highly depleted. A probable situation is shown in Fig. 1(iii) where many of the misplaced $\mathrm{Ru}^{4+}$ ions can still manage to interact with other $\mathrm{Ru}^{4+}$ ions forming clusters,
Table I: The lattice constants of $\mathrm{Ba}_{3} \mathrm{RuTi}_{2} \mathrm{O}_{9}$ obtained from our refinement are compared with literature data.

\begin{tabular}{|c|c|c|c|c|}
\hline Material & Space group & a $(\AA)$ & c $(\AA)$ & Source \\
\hline \hline $\mathrm{Ba}_{3} \mathrm{RuTi}_{2} \mathrm{O}_{9}$ & $\mathrm{P} 6_{3} m c$ & $5.7204(3)$ & $14.0109(3)$ & this work \\
\hline $\mathrm{Ba}_{3} \mathrm{RuTi}_{2} \mathrm{O}_{9}$ & $\mathrm{P} 6_{3} m c$ & 5.7056 & 14.0093 & Ref. [13] \\
\hline $\mathrm{Ba}_{3} \mathrm{IrTi}_{2} \mathrm{O}_{9}$ & $\mathrm{P} 6_{3} m c$ & 5.7214 & 14.0721 & Ref. [9] \\
\hline $\mathrm{BaTiO}_{3}$ & $\mathrm{P} 6_{3} / m m c$ & 5.7238 & 13.9649 & Ref. [14] \\
\hline
\end{tabular}

Table II: The atomic parameters obtained by refining x-ray powder diffraction data for $\mathrm{Ba}_{3} \mathrm{RuTi}_{2} \mathrm{O}_{9}$ at room temperature with the space group $\mathrm{P} 6{ }_{3} \mathrm{mc}$.

\begin{tabular}{|c|c|c|c|c|c|}
\hline \multicolumn{2}{|c|}{} & $\mathrm{x}$ & $\mathrm{y}$ & $\mathrm{z}$ & $\mathrm{g}$ \\
\hline $\mathrm{Ba}(1)$ & $2 \mathrm{a}$ & 0 & 0 & $0.249(3)$ & 1.00 \\
\hline $\mathrm{Ba}(2)$ & $2 \mathrm{~b}$ & $1 / 3$ & $2 / 3$ & $0.085(3)$ & 1.00 \\
\hline $\mathrm{Ba}(3)$ & $2 \mathrm{~b}$ & $1 / 3$ & $2 / 3$ & $0.397(3)$ & 1.00 \\
\hline $\mathrm{Ru}(1)$ & $2 \mathrm{~b}$ & $1 / 3$ & $2 / 3$ & $0.651(3)$ & $0.61(9)$ \\
\hline $\mathrm{Ti}(1)$ & $2 \mathrm{~b}$ & $1 / 3$ & $2 / 3$ & $0.651(3)$ & $0.39(9)$ \\
\hline $\mathrm{Ti}(2)$ & $2 \mathrm{~b}$ & $1 / 3$ & $2 / 3$ & $0.839(3)$ & $0.69(9)$ \\
\hline $\mathrm{Ru}(2)$ & $2 \mathrm{~b}$ & $1 / 3$ & $2 / 3$ & $0.839(3)$ & $0.31(9)$ \\
\hline $\mathrm{Ti}(3)$ & $2 \mathrm{a}$ & 0 & 0 & $0.510(3)$ & $0.92(4)$ \\
\hline $\mathrm{Ru}(3)$ & $2 \mathrm{a}$ & 0 & 0 & $0.510(3)$ & $0.08(4)$ \\
\hline $\mathrm{O}(1)$ & $6 \mathrm{c}$ & $0.160(3)$ & $0.838(3)$ & $0.575(3)$ & 1.00 \\
\hline $\mathrm{O}(2)$ & $6 \mathrm{c}$ & $0.488(4)$ & $0.511(4)$ & $0.749(4)$ & 1.00 \\
\hline $\mathrm{O}(3)$ & $6 \mathrm{c}$ & $0.160(3)$ & $0.838(3)$ & $0.915(3)$ & 1.00 \\
\hline
\end{tabular}

while some, called orphan spins, remain isolated.

Further we have measured susceptibility of the material at different fields as shown in Fig. 3, No long range magnetic ordering down to $2 \mathrm{~K}$ is found whereas a splitting between $\mathrm{ZFC}$ and $\mathrm{FC}$ data at $30 \mathrm{~K}$ is seen which may be indicative of a transition to a frozen magnetic state. With increasing field, the splitting becomes less prominent and vanishes at an applied field of $10 \mathrm{kOe}$. We have fitted our susceptibility data with the CurieWeiss (CW) formula $\chi=\chi_{0}+C /(T-\theta)$ in the temperature range $100-350 \mathrm{~K}$ (shown in Fig. (4) which yields the temperature independent susceptibility $\chi_{0}=2.05 \times$ $10^{-4} \mathrm{~cm}^{3} / \mathrm{mol}$, Curie constant $C=0.7 \mathrm{~cm}^{3} \mathrm{~K} / \mathrm{mol}$ and $\theta_{\mathrm{CW}}=-166 \mathrm{~K}$. For insulating oxides one can consider $\chi_{0}=\chi_{c o r e}+\chi_{v v}$, where $\chi_{\text {core }}$ is the core diamagnetic susceptibility and $\chi_{v v}$ is Van-Vleck paramagnetic susceptibility. For our sample $\chi_{\text {core }}=-2.32 \times 10^{-4} \mathrm{~cm}^{3} / \mathrm{mol}$ [15], which results in $\chi_{v v}=4.37 \times 10^{-4} \mathrm{~cm}^{3} / \mathrm{mol}$. This value of $\chi_{v v}$ is similar to that found in other $\mathrm{Ru}$ based insulating oxides like $\mathrm{La}_{2} \mathrm{RuO}_{5}\left[16\right.$ and $\mathrm{La}_{2} \mathrm{LiRuO}_{6}$ [17]. Inverse susceptibility of the material is shown on the right axis of Fig. 目 (after subtracting the temperature independent part $\chi_{0}$ ) which is linear with temperature in a wide range of temperature and deviates from linearity below $T \sim 80 \mathrm{~K}$.

We now compare our susceptibility data with those of Ref. 11 by Byrne et al. A slope change was found by Byrne et al. in the inverse susceptibility of $\mathrm{Ba}_{3} \mathrm{RuTi}_{2} \mathrm{O}_{9}$ 


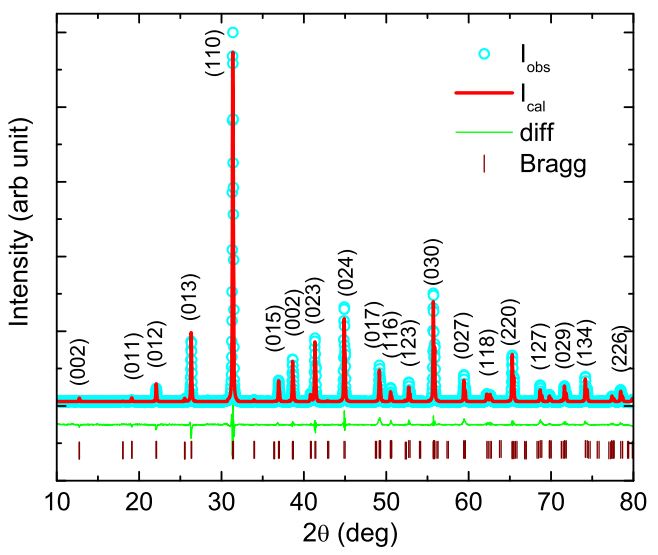

Figure 2: The x-ray diffraction pattern of $\mathrm{Ba}_{3} \mathrm{RuTi}_{2} \mathrm{O}_{9}$ is shown. The refinement of these data using the space group $\mathrm{P} 6_{3} m c$ is also shown. Light blue circles indicate experimental pattern and the red line indicates the theoretical pattern while the green line is the difference between the two. The Bragg positions are shown as vertical lines. The (hkl) planes corresponding to the peaks are also marked in the figure.

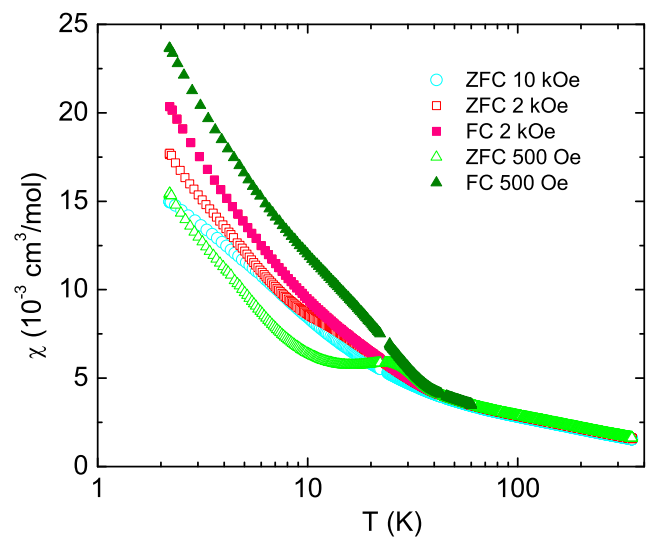

Figure 3: The magnetic susceptibility of $\mathrm{Ba}_{3} \mathrm{RuTi}_{2} \mathrm{O}_{9}$ at different fields is shown as a function of temperature in semi-log scale. Solid symbols represent FC and open symbols represent ZFC measurements.

at $150 \mathrm{~K}$ which is absent in our data. Moreover, they found $\theta_{\mathrm{CW}}=-256 \mathrm{~K}$ and $\mu_{\text {eff }}=2.86 \mu_{B}$ which are higher than $\theta_{\mathrm{CW}}=-166 \mathrm{~K}$ and $\mu_{\text {eff }}=2.37 \mu_{B}$ obtained from our analysis. To clarify this discrepancy, we have reanalysed the published data as shown in Fig. 4. The green, open triangles represent the inverse susceptibility data as published in Ref. [11]. The corresponding susceptibility is shown by blue circles on the left axis and fitted by us with the CW law in the range $118-335 \mathrm{~K}$. This fitting gives the temperature independent susceptibility $\chi_{0 \mathrm{~B}}=5.55 \times 10^{-4} \mathrm{~cm}^{3} / \mathrm{mol}, C=0.54 \mathrm{~cm}^{3} \mathrm{~K} / \mathrm{mol}$

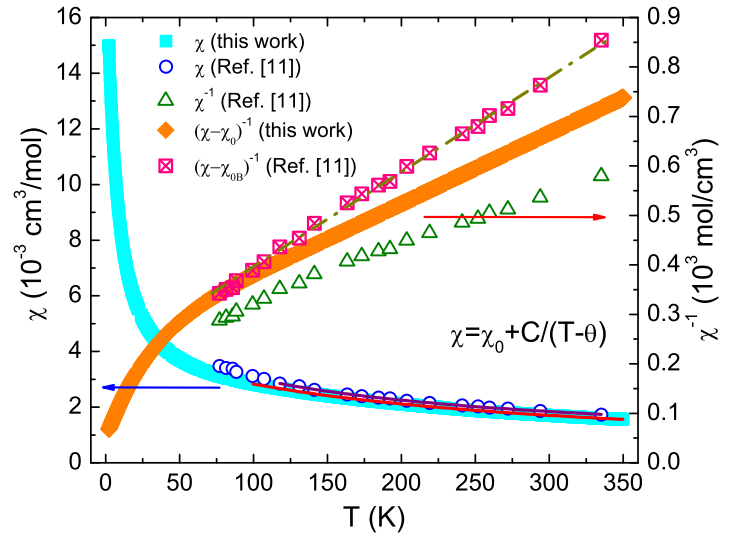

Figure 4: The magnetic susceptibility of $\mathrm{Ba}_{3} \mathrm{RuTi}_{2} \mathrm{O}_{9}$ and its fitting with the Curie-Weiss law (red solid line) is shown. The inverse susceptibility (after subtracting $\chi_{0}$ ) is shown with orange diamonds. The inverse susceptibility data of $\mathrm{Ba}_{3} \mathrm{RuTi}_{2} \mathrm{O}_{9}$ obtained from Ref. [11] is shown as green triangles. The corresponding susceptibility is shown as blue circles with its fit with CW law (violet solid line). The inverse susceptibility after subtracting $\chi_{0 \mathrm{~B}}$ (obtained from fit, see text) is also shown with pink squares. The dashed line is a guide to eye. All the susceptibilities are plotted on the left axis while the inverse susceptibilities correspond to the right axis. The solid symbols denote the data from this work and open symbols are used for data from Ref. [11].

$\left(\mu_{e f f}=2.08 \mu_{B}\right)$ and $\theta_{\mathrm{CW}}=-116 \mathrm{~K}$. These values are somewhat closer to the values obtained by us. Subtracting this $\chi_{0 \mathrm{~B}}$, we have plotted $\left(\chi-\chi_{0 \mathrm{~B}}\right)^{-1}$ which is linear in the whole temperature range and the kink at $150 \mathrm{~K}$ is also absent. Apparently, Byrne et al. did not use the correct temperature-independent susceptibility, leading them to infer an incorrect Curie term. Also, they calculated $\mu_{\text {eff }}$ based on a single susceptibility data point (one temperature) which certainly can mislead.

The $C$ value obtained from the $\mathrm{CW}$ fit of our data is almost $70 \%$ of the paramagnetic $S=1$ value. Normally in $3 d$ transition metal oxides the $C$ value turns out as expected from a pure spin contribution. But for $5 d$ transition metal oxides the $C$ value obtained is normally much smaller than expected from a pure spin contribution. In case of $\mathrm{Ba}_{3} \mathrm{IrTi}_{2} \mathrm{O}_{9}$ this was only $40 \%$ of the expected spin-only value [9]. For $4 d$ based systems the impact of the orbital effect is expected to be in between that in $3 d$ and $5 d$ materials. Hence the reduction in $C$ obtained from our analysis is most likely an effect of spinorbit coupling present in the system. A high $(-166 \mathrm{~K})$ $\theta_{\mathrm{CW}}$ obtained from $\mathrm{CW}$ fit confirms that the $\mathrm{Ru}^{4+}$ spins are strongly correlated. Probably a large site sharing is responsible in forming some magnetic clusters which give rise to frozen moments below $30 \mathrm{~K}$.

The heat capacity $\left(C_{\mathrm{P}}\right)$ of a material gives prominent signatures pertaining to any structural or magnetic transitions. We have measured $C_{\mathrm{P}}$ at different fields as shown 


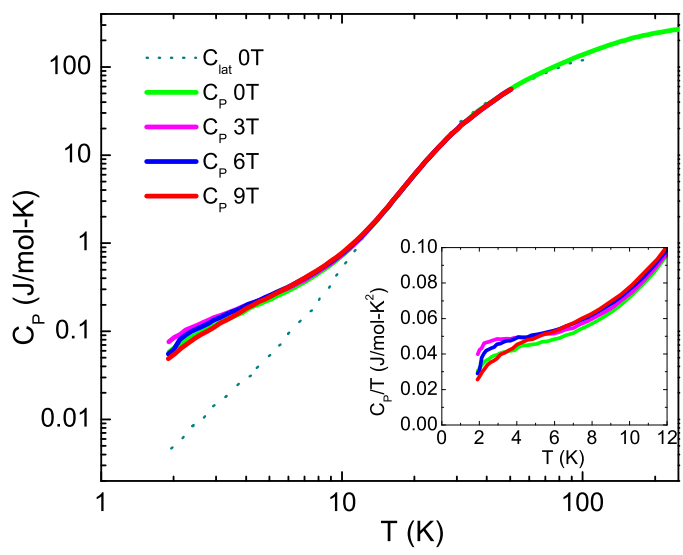

Figure 5: The heat capacity $C_{\mathrm{P}}$ of $\mathrm{Ba}_{3} \mathrm{RuTi}_{2} \mathrm{O}_{9}$ measured at different fields is shown in a log-log scale. The lattice heat capacity (taken from Ref. [20]) is also shown. Inset: $C_{\mathrm{P}} / T$ is shown as a function of $T$ in the low- $T$ region.

in Fig. 5. No anomaly is found in our $C_{\mathrm{P}}$ measurement in the $T$ range $1.8 \mathrm{~K} \leq T \leq 295 \mathrm{~K}$.

The total heat capacity of the sample has three contributions namely, (i) lattice contribution $\left(C_{\text {lat }}\right)$ (ii) magnetic contribution $\left(C_{\mathrm{M}}\right)$ and (iii) Schottky contribution $\left(C_{\mathrm{Sch}}\right)$ from orphan spins. The nuclear Schottky anomaly normally appears at much lower temperature and probably will not affect our data which is down to $1.8 \mathrm{~K}$ only. To extract the magnetic heat capacity we have to subtract out the $C_{\text {lat }}$ and $C_{\text {Sch }}$ from the total heat capacity. We now attempt to estimate the magnetic contribution by subtracting the other two parts.

The generalised heat capacity expression for two level Schottky anomaly is [18, 19],

$$
C_{\mathrm{Sch}}(\Delta)=R \frac{g_{0}}{g_{1}}\left(\frac{\Delta}{k_{B} T}\right)^{2} \frac{\exp \left(\frac{\Delta}{k_{B} T}\right)}{\left[1+\frac{g_{0}}{g_{1}} \exp \left(\frac{\Delta}{k_{B} T}\right)\right]^{2}}
$$

where $\Delta$ is the level splitting, $R$ is the universal gas constant, $k_{B}$ is the Boltzman constant and $g_{0}$ and $g_{1}$ are the degeneracies of ground state and excited state, respectively. For spin $S=1\left(\mathrm{Ru}^{4+}\right)$ systems, Eq. 1 can be modified with the approximation as $g_{0}=1$ and $g_{1}=2$ [18].

We have subtracted $C_{\mathrm{P}}(0 \mathrm{~T})$ from $C_{\mathrm{P}}(9 \mathrm{~T})$ data and further divided that by temperature. Any lattice contribution is therefore removed by this procedure. The $\left[C_{\mathrm{P}}(9 \mathrm{~T})-C_{\mathrm{P}}(0 \mathrm{~T})\right] / T$ data are shown in the inset of Fig. 66 with its fit to $f\left[C_{\mathrm{Sch}}\left(\Delta_{9 \mathrm{~T}}\right)-C_{\mathrm{Sch}}\left(\Delta_{0 \mathrm{~T}}\right)\right] / T$. In this expression, $f$ is the fraction of orphan spins present in the sample and $C_{\mathrm{Sch}}\left(\Delta_{9 \mathrm{~T}}\right)$, and $C_{\mathrm{Sch}}\left(\Delta_{0 \mathrm{~T}}\right)$ are the Schottky anomalies corresponding to the level splittings of $\Delta_{9 \mathrm{~T}}$ and $\Delta_{0 \mathrm{~T}}$ with applied magnetic fields $9 \mathrm{~T}$ and $0 \mathrm{~T}$, respectively. The fit is reasonably good and suggests that the

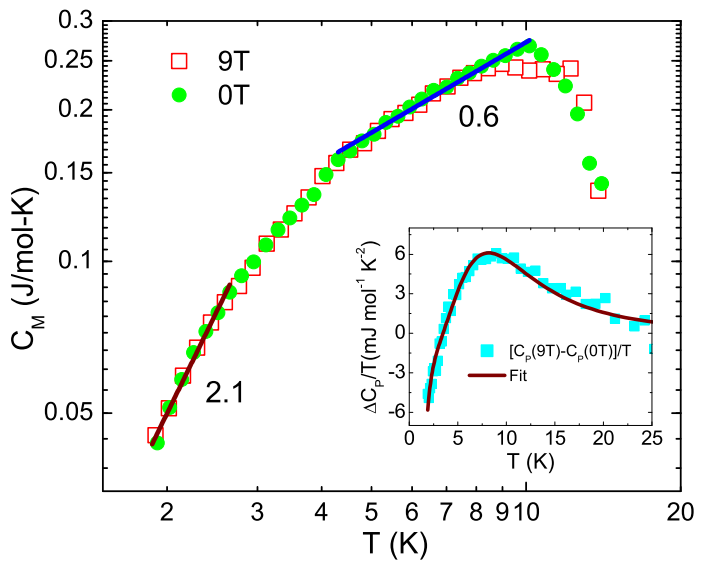

Figure 6: The magnetic heat capacity data of $\mathrm{Ba}_{3} \mathrm{RuTi}_{2} \mathrm{O}_{9}$ at $0 \mathrm{~T}$ and $9 \mathrm{~T}$ are shown in a log-log scale. The solid lines show power law fits with temperature with the power shown in figure. Inset: $\left[C_{\mathrm{P}}(9 \mathrm{~T})-C_{\mathrm{P}}(0 \mathrm{~T})\right] / T$ and its fit with Schottky model (solid line) is shown.

field dependence of $C_{\mathrm{P}}$ is coming from $C_{\mathrm{Sch}}$ only. From the fit we found $\Delta_{9 \mathrm{~T}} / k_{B}=27.7 \mathrm{~K}, \Delta_{0 \mathrm{~T}} / k_{B}=1.5 \mathrm{~K}$ and $\sim 1 \%$ orphan spins are present in the system. Putting the corresponding $\Delta$ (obtained from fitting) in Eq. 11]and multiplying by $f$, we can get the Schottky contribution $\left(C_{\mathrm{Sch}}\right)$ to the heat capacity for a particular field.

To subtract the lattice contribution $\left(C_{\text {lat }}\right)$ from the total heat capacity $\left(C_{\mathrm{P}}\right)$ one needs a non-magnetic ana$\log$ of the system. Unfortunately, we didn't find a good non-magnetic analog of $\mathrm{Ba}_{3} \mathrm{RuTi}_{2} \mathrm{O}_{9}$. However we have earlier used $\mathrm{Ba}_{3} \mathrm{ZnSb}_{2} \mathrm{O}_{9}$ (taken from Ref. [20]) as a non-magnetic analog in the case of $\mathrm{Ba}_{3} \mathrm{IrTi}_{2} \mathrm{O}_{9}$ [9]. $\mathrm{Ba}_{3} \mathrm{ZnSb}_{2} \mathrm{O}_{9}$ can be used as a non magnetic analog in the present case as well since both have similar crystal structure. The heat capacities of these two samples have a mismatch at higher temperature due to the difference in their molecular mass and volume. We have used a multification factor of $\sim 0.67$ so that $C_{P}$ data of $\mathrm{Ba}_{3} \mathrm{ZnSb}_{2} \mathrm{O}_{9}$ matches with that of $\mathrm{Ba}_{3} \mathrm{RuTi}_{2} \mathrm{O}_{9}$ in the nominal temperature range $40-60 \mathrm{~K}$ (shown in Fig. 5). The corrected heat capacity of $\mathrm{Ba}_{3} \mathrm{ZnSb}_{2} \mathrm{O}_{9}$ can be considered as the lattice contribution $\left(C_{\text {lat }}\right)$ to the total heat capacity of $\mathrm{Ba}_{3} \mathrm{RuTi}_{2} \mathrm{O}_{9}$. This $C_{\text {lat }}$ is subtracted from $C_{\mathrm{P}}$ and we are left with the $C_{\mathrm{M}}$ and $C_{\mathrm{Sch}}$. Further we have subtracted $C_{\text {Sch }}$ obtained from our previous analysis and finally obtained $C_{\mathrm{M}}$.

The $C_{\mathrm{M}}$ is independent of field as shown in Fig. 6 and follows a power law in temperature with power 0.6 in the range $4.3-10 \mathrm{~K}$ and with power 2.1 below $2.7 \mathrm{~K}$. This suggests that spin excitations are present in this system. We have also estimated the entropy change $\triangle \mathrm{S}_{\mathrm{M}}=0.36 \mathrm{~J} / \mathrm{mol}-\mathrm{K}$ by integrating $C_{\mathrm{M}} / T$. This value is much smaller than the expected value of $R \ln (2 S+1)$ $=9.13 \mathrm{~J} / \mathrm{mol}-\mathrm{K}$ for a spin-only system. This reduction 
could be partly due to short-range magnetic ordering taking place in the system starting at high temperatures and the entropy is released over a large range of temperature. In addition, a strong spin-orbit coupling suppressing the magnetic moments could also be responsible. Whereas other reasons like error in estimation of $C_{\text {lat }}$ due to lack of a proper non-magnetic analog may also play a role, it appears that a majority of spins remain disordered and do not freeze.

\section{CONCLUSIONS}

We have prepared $\mathrm{Ba}_{3} \mathrm{RuTi}_{2} \mathrm{O}_{9}$ (a $4 d$ analog of the $5 d$ system $\mathrm{Ba}_{3} \mathrm{IrTi}_{2} \mathrm{O}_{9}$ ) and measured its magnetic susceptibility and heat capacity at different fields. No long range magnetic ordering is found down to $1.8 \mathrm{~K}$ despite a large $\theta_{\mathrm{CW}}$ obtained from $\mathrm{CW}$ fitting of susceptibility data. This indicates that the spins are strongly correlated and the system is highly frustrated. The Curie constant is about $30 \%$ smaller than that expected for isolated $S=1$ moments. This might be because of a spin-orbit coupling present in this system. Splitting of ZFC and FC susceptibility data at $30 \mathrm{~K}$ indicates formation of a frozen magnetic phase. Magnetic heat capacity of this material is independent of field and approaches a power law at low temperature with power 2.1. These measurements suggest that $\mathrm{Ba}_{3} \mathrm{RuTi}_{2} \mathrm{O}_{9}$ is a highly frustrated system but the nature of its ground state is not very clear. Local probe investigations like $\mu \mathrm{SR}$ could be useful to check if the spin freezing happens in the bulk of the sample or not.

\section{ACKNOWLEDGEMENT}

We acknowledge the financial support from Indo-Swiss Joint Research Program, Department of Science and Technology, India. We thank K. Prsa for his help in some supporting measurements and H. M. Ronnow for useful discussions.
[1] B. J. Kim, H. Jin, S. J. Moon, J.-Y. Kim, B.-G. Park, C. S. Leem, Jaejun Yu, T.W. Noh, C. Kim, S.-J. Oh, J.-H. Park, V. Durairaj, G. Cao, and E. Rotenberg, Phys. Rev. Lett. 101, 076402 (2008)

[2] B. J. Kim, H. Ohsumi, T. Komesu, S. Sakai, T. Morita, H. Takagi, and T. Arima, Science 323, 1329 (2009)

[3] M. W. Haverkort, I. S. Elfimov, L. H. Tjeng, G. A. Sawatzky, and A. Damascelli, Phys. Rev. Lett. 101, 026406 (2008)

[4] T. Mizokawa, L. H. Tjeng, G. A. Sawatzky, G. Ghiringhelli, O. Tjernberg, N. B. Brookes, H. Fukazawa, S. Nakatsuji, and Y. Maeno, Phys. Rev. Lett. 87, 077202 (2001)

[5] Y. Singh and P. Gegenwart, Phys. Rev. B 82, 064412 (2010)

[6] Y. Okamoto, M. Nohara, H. Aruga-Katori, and H. Takagi, Phys. Rev. Lett. 99, 137207 (2007)

[7] R. S. Perry, L. M. Galvin, S. A. Grigera, L. Capogna, A. J. Schofield, A. P. Mackenzie, M. Chiao, S. R. Julian, S. Ikeda, S. Nakatsuji, Y. Maeno, and C. Pfleiderer, Phys. Rev. Lett. 86, 2661 (2001)

[8] G. Cao, X. N. Lin, L. Balicas, S. Chikara, J. E. Crow, and P. Schlottmann, New J. Phys. 6, 159 (2004)

[9] T. Dey, A. V. Mahajan, P. Khuntia, M. Baenitz, B. Koteswararao, and F. C. Chou, Phys. Rev. B 86, 140405(R) (2012)

[10] J. G. Dickson, L. Katz, and R. Ward, J. Amer. Chem. Soc. 83, 3026 (1961)

[11] R. C. Byrne, and C. W. Moeller, J. Solid State Chem. 2,
$228(1970)$

[12] G. Radtke, C. Maunders, A. Saúl, S. Lazar, H. J. Whitfield, J. Etheridge, and G. A. Botton, Phys. Rev. B 81, 085112 (2010)

[13] C. Maunders, J. Etheridge, N. Wright, and H. J. Whitfield, Acta Crystallogr., Sect. B: Struct. Sci. 61, 154 (2005)

[14] J. Akimoto, Y. Gotoh, and Y. Oosawa, Acta Cryst. C 50, 160 (1994)

[15] P. W. Selwood, Magnetochemistry (Interscience, New York, 1956)

[16] S. Riegg, A. Günther, H.-A. K. von Nidda, A. Loidl, M. V. Eremin, A. Reller, and S. G. Ebbinghaus, Phys. Rev. B 86, 115125 (2012)

[17] T. Aharen, J. E. Greedan, F. Ning, T. Imai, V. Michaelis, S. Kroeker, H. Zhou, C. R. Wiebe, and L. M. D. Cranswick, Phys. Rev. B 80, 134423 (2009)

[18] E. S. R. Gopal, Specific Heats at Low Temperatures Plenum, New York, 1966

[19] C. He, H. Zheng, J. F. Mitchell, M. L. Foo, R. J. Cava, and C. Leighton, Appl. Phys. Lett. 94, 102514 (2009)

[20] H. D. Zhou, E. S. Choi, G. Li, L. Balicas, C. R. Wiebe, Y. Qiu, J. R. D. Copley, and J. S. Gardner, Phys. Rev. Lett. 106, 147204 (2011) and Supplemental Material of J. G. Cheng, G. Li, L. Balicas, J. S. Zhou, J. B. Goodenough, C. Xu, and H. D. Zhou, Phys. Rev. Lett. 107, 197204 (2011) 